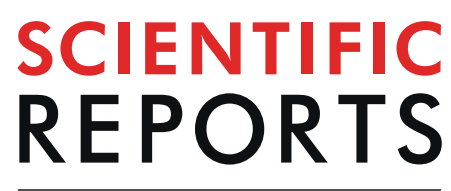

natureresearch

\title{
OPEN Multiplex coherent anti-Stokes Raman scattering highlights state of chromatin condensation in $\mathrm{CH}$
}

Received: 16 May 2019

Accepted: 6 August 2019

Published online: 25 September 2019

\section{region}

\author{
Tiffany Guerenne-Del Ben ${ }^{1}$, Zakaniaina Rajaofara ${ }^{2}$, Vincent Couderc ${ }^{2}$, Vincent Sol ${ }^{1}$, \\ Hideaki Kano ${ }^{3,4}$, Philippe Leproux $\mathbb{1}^{2,5}$ \& Jean-Michel Petit ${ }^{1}$
}

Coherent Raman microscopy has become a powerful tool in label-free, non-destructive and fast cell imaging. Here we apply high spectral resolution multiplex coherent anti-Stokes Raman scattering (MCARS) microspectroscopy in the high wavenumber region to the study of the cell cycle. We show that heterochromatin - the condensed state of chromatin - can be visualised by means of the vibrational signature of proteins taking part in its condensation. Thus, we are able to identify chromosomes and their movement during mitosis, as well as structures like nucleoli and nuclear border in interphase. Furthermore, the specific organization of the endoplasmic reticulum during mitosis is highlighted. Finally, we stress that MCARS can reveal the biochemical impact of the fixative method at the cellular level. Beyond the study of the cell cycle, this work introduces a label-free imaging approach that enables the visualization of cellular processes where chromatin undergoes rearrangements.

In the last two decades, Raman microspectroscopy has become a powerful tool in label-free cell imaging. It could for example reveal cellular components in neutrophils, such as a multilobed nucleus, lipid droplets, nucleoli and mitochondria ${ }^{1}$. It made also possible to identify the endoplasmic reticulum, the Golgi apparatus and intracellular vesicles in HeLa cells ${ }^{2}$. Basically, such identifications are realised by means of the vibrational signature of lipids and proteins. However the sole lipid signature could be used to investigate cell lines such as cancer cells ${ }^{3,4}$ and to differentiate proliferating cells from senescent ones through modifications of the nuclear envelope $\mathrm{e}^{5}$. Recently Raman microspectroscopy was applied to evaluate the impact of an anti-cancer treatment ${ }^{6}$.

On the other hand, Raman imaging has some drawbacks that limit its dissemination. Namely, the vibrational signal overlaps with autofluorescence, which is inherent in biological samples. This results in a reduction of the signal-to-noise ratio. In connection with this aspect and due to the spontaneous nature of Raman scattering, the analysis time remains long and is generally not consistent with the observation of living cells. To avoid this limitation, the use of coherent anti-Stokes Raman scattering (CARS) microscopy is particularly appropriate. Since the demonstration of its high sensitivity and high spatial resolution in $1999^{7}$, CARS microscopy has been extensively employed to image prokaryotes, mammal and plant cells, human tissues, etc. Commonly, CARS technology is used to analyse lipid content in cells through the strong vibrational signature of methylene $\left(\mathrm{CH}_{2}\right)$ groups at $2850 \mathrm{~cm}^{-17,8}$. Using this signature, authors could monitor the differentiation of fetal skeletal stem cells in adipogenic or chondrogenic cells ${ }^{9}$, the uptake of lipids in monocytes ${ }^{10}$ or in fibroblasts ${ }^{11}$. Others characterized murine stem cells during their differentiation into adipocytes ${ }^{12}$. A link between lipid-rich tumour cells and their aggressive comportment was established by CARS analysis ${ }^{13}$. More specifically, it was possible to identify circulating tumour cells using the specific signature of lipids ${ }^{14}$.

In a CARS experiment, the sample is illuminated with two laser waves simultaneously, namely the pump $\left(\omega_{\mathrm{p}}\right.$ frequency) and Stokes ( $\omega_{\mathrm{s}}$ frequency) waves. When the difference $\omega_{\mathrm{p}}-\omega_{\mathrm{S}}$ is equal to the vibration frequency of

${ }^{1}$ PEIRENE, EA 7500, University of Limoges, 123 avenue Albert Thomas, 87060, Limoges, France. ${ }^{2}$ XLIM, UMR 7252, University of Limoges, 123 avenue Albert Thomas, 87060, Limoges, France. ${ }^{3}$ Department of Applied Physics, Graduate School of Pure and Applied Sciences, University of Tsukuba, 1-1-1 Tennodai, Tsukuba, Ibaraki, 305-8573, Japan. ${ }^{4}$ Institute of Applied Physics, University of Tsukuba, 1-1-1 Tennodai, Tsukuba, Ibaraki, 305-8573, Japan. ${ }^{5}$ LEUKOS, 37 rue Henri Giffard, 87280, Limoges, France. Correspondence and requests for materials should be addressed to P.L. (email: philippe.leproux@unilim.fr) or J.-M.P. (email: jean-michel.petit@unilim.fr) 
the molecular bond of interest, the resonant CARS signal is generated at frequency $2 \omega_{p}-\omega_{s}$. CARS being a coherent process, the signal intensity can be strongly enhanced compared to spontaneous Raman scattering, allowing to reduce the analysis time $\mathrm{e}^{9,15}$.

In order to generate the resonant CARS signal over the full range of Raman shifts (roughly $500-3500 \mathrm{~cm}^{-1}$, including both the fingerprint, $\mathrm{CH}$ and $\mathrm{OH}$ stretching regions) simultaneously, it is necessary to use a Stokes wave having a very broad spectrum at wavelengths longer than that of the pump. Such radiation can be obtained by generating a supercontinuum in a photonic crystal fibre $(\mathrm{PCF})^{16}$. By this way, and by ensuring the synchronicity between the pump and Stokes pulse trains, it is possible to implement so-called "multiplex CARS" (MCARS) $)^{17,18}$ or "broadband CARS" (BCARS) ${ }^{19,20}$ microspectroscopy. These configurations can then provide rich vibrational information from biological samples.

To date CARS microscopy/microspectroscopy has been massively applied to the label-free imaging of fixed or living cells, highlighting its ability to discern intracellular constituents such as nucleus, nucleoli, mitochondria, etc. However, these studies generally do not take into account the fact that proliferating cells in vitro constitute a heterogeneous cell population, mainly due to cell cycle ${ }^{21}$, during which all cell types change their DNA, RNA, lipid and protein contents. During cell cycle, the cells pass through four distinct phases: $G_{1}, S, G_{2}$ and $M$. $G_{1}$ and $\mathrm{G}_{2}$ phases are characterized by protein and RNA syntheses in order to prepare cells to $S$ phase (DNA synthesis) then $\mathrm{M}$ phase (mitosis) ${ }^{22}$ respectively. Moreover, the location and organisation of chromatin differs according to $\mathrm{M}$ sub-phases: (1) during prophase the chromosomes become observable due to a higher level of chromatin condensation, and the nuclear envelope dissociates; (2) the prometaphase is characterized by the chromosome capture by microtubules, and by the organization of mitotic spindle; (3) in metaphase chromosomes are aligned at the metaphase plate; (4) during anaphase, the sister chromatids of each chromosome separate; (5) telophase corresponds to the end of mitosis with chromatin decondensation and nuclear envelope restructuration; cytokinesis marks the end of cell division.

Since the work by Matthäus et al. in 2006 based on spontaneous Raman scattering ${ }^{23}$, very few studies of cell cycle have been realised by means of coherent Raman microscopy. In 2010, CARS imaging of proteins $\left(2928 \mathrm{~cm}^{-1}\right)$ and lipids $\left(2840 \mathrm{~cm}^{-1}\right)$ in live mitotic and interphase cells was done, but no correlation was observed between the DNA compaction level and the proteins' signal ${ }^{24}$. Recently, cell division (prometaphase, anaphase, telophase) was investigated by using CARS in the $\mathrm{CH}$ stretching region, as well, and by applying a cumbersome data analysis method rendering images of water, proteins, DNA/proteins and lipids ${ }^{25}$. The reproducibility of the study was considered by merely analysing a second prometaphase cell. Moreover, this study is somewhat reminiscent of a previous work where linear decomposition of multicolour stimulated Raman scattering (SRS) data was used to image DNA in live mitotic and interphase cells ${ }^{26}$. Finally, MCARS with $600-3200 \mathrm{~cm}^{-1}$ coverage could be used to achieve molecular fingerprinting of living cells at different phases of the cell cycle ${ }^{27}$. Such spectral coverage includes many vibrational bands in the fingerprint and $\mathrm{CH}$ stretching regions with selectivity to DNA/RNA, proteins and lipids.

In this work, we apply MCARS microspectroscopy in the $\mathrm{CH}$ stretching vibrational region $\left(2500-3200 \mathrm{~cm}^{-1}\right)$ with high spectral resolution $\left(<1 \mathrm{~cm}^{-1}\right)$ to the study of cell cycle, including a high number of fixed and living cells in interphase and all sub-phases of mitosis. We show that, throughout the cell cycle, heterochromatin - the condensed state of chromatin - can be visualised in the $\mathrm{CH}_{3}$ stretching band by means of the vibrational signature of proteins that take part in its condensation. This allows, in particular, localising chromosomes in mitotic cells. Significant spatial discrimination is demonstrated between the $\mathrm{CH}_{3}$ signal, predominantly localised in the nucleus, and the $\mathrm{CH}_{2}$ signal that highlights a greater quantity of lipids in the cytoplasm. Moreover, we look into the impact of cell fixation using paraformaldehyde (PFA) on the vibrational signature, with living cells as a reference. This work shows that high spectral resolution MCARS in the $\mathrm{CH}$ region is a user-friendly, label-free method enabling the observation of DNA/protein/lipid content with high contrast and short time, suitable for the analysis of living cells.

\section{Results}

Verification of cell cycle distribution and morphology. Figure 1 displays the results of flow cytometry analysis for different HEK293 cell cultures. For asynchronous culture, $46 \%$ of cells were in $\mathrm{G}_{1}$ phase, $46 \%$ in S phase and only $8 \%$ in $\mathrm{G}_{2} / \mathrm{M}$ phases (Fig. 1a). After double thymidine block, a large number of cells (68\%) was in $\mathrm{G}_{1}$ phase, versus $32 \%$ in $\mathrm{S}$ phase (Fig. $1 \mathrm{~b}$ ). As a matter of fact, the thymidine treatment is unable to block all cells at the $\mathrm{G}_{1} / \mathrm{S}$ boundary, but it can be concluded that all cells were in interphase. After thymidine and nocodazole treatment, $70 \%$ of cells were arrested in $M$ phase (Fig. 1c).

Furthermore, we analysed cell cultures after DAPI staining by means of bright-field and fluorescence microscopies, which allow visualising the morphology of cells and nuclei, respectively (Fig. 1d). For interphase $\left(G_{1} / S\right)$ cells, a typical spread morphology is observed; the nucleus is well delimitated, and its staining is heterogeneous. In contrast, mitotic cells exhibit a preferably round shape $\mathrm{e}^{28}$; the staining reveals the presence of hypercondensed chromatin (heterochromatin), which contributes to chromosome formation. Moreover, the overall higher fluorescence intensity is characteristic of an abundant binding of DAPI to more condensed DNA. It is noticeable that cells arrested in M phase are prophase cells.

Analysis of fixed cells in interphase $\left(G_{1} / S\right)$ and mitosis (prophase). Figure 2 illustrates the experimental and numerical methodology used for analysing HEK293 cell cultures. First, cells of interest were selected using bright-field and/or fluorescence imaging, depending on whether or not they were stained with DAPI. Then selected cells were mapped by means of high spectral resolution $\left(<1 \mathrm{~cm}^{-1}\right)$ MCARS microspectroscopy in the $2500-3200 \mathrm{~cm}^{-1}$ range. Finally, the vibrational information was extracted by using the maximum entropy method $(\mathrm{MEM})^{29}$, and cell images were reconstructed at $2850 \mathrm{~cm}^{-1}\left(\mathrm{CH}_{2}\right.$ symmetric stretching) and $2930 \mathrm{~cm}^{-1}\left(\mathrm{CH}_{3}\right.$ symmetric stretching). 
a

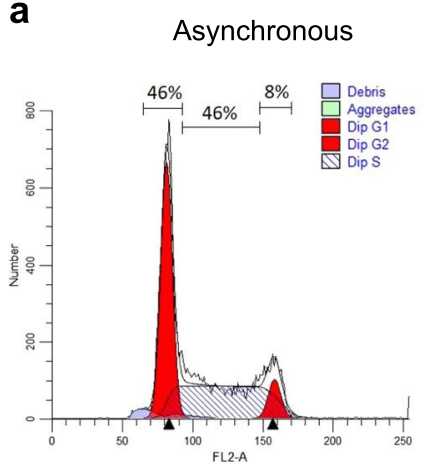

b

Interphase $\left(G_{1} / S\right)$

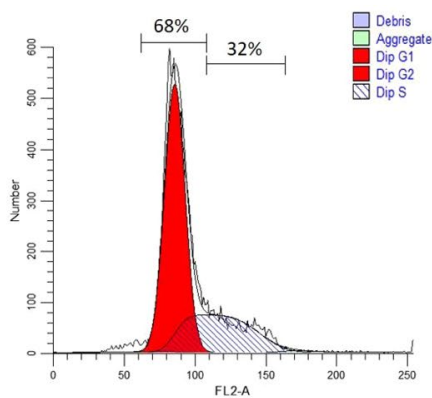

C

Mitosis (prophase)

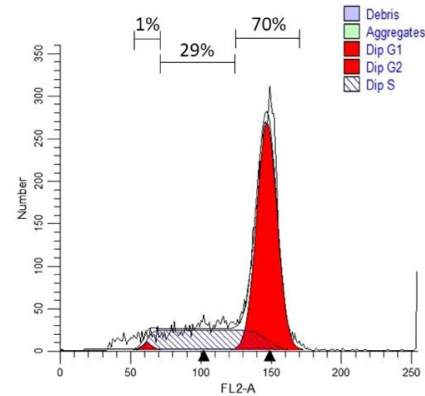

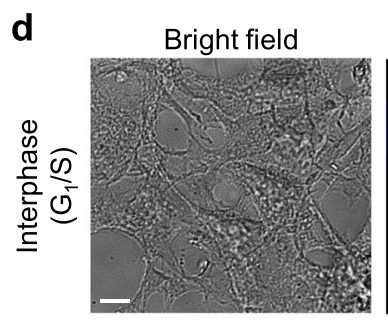
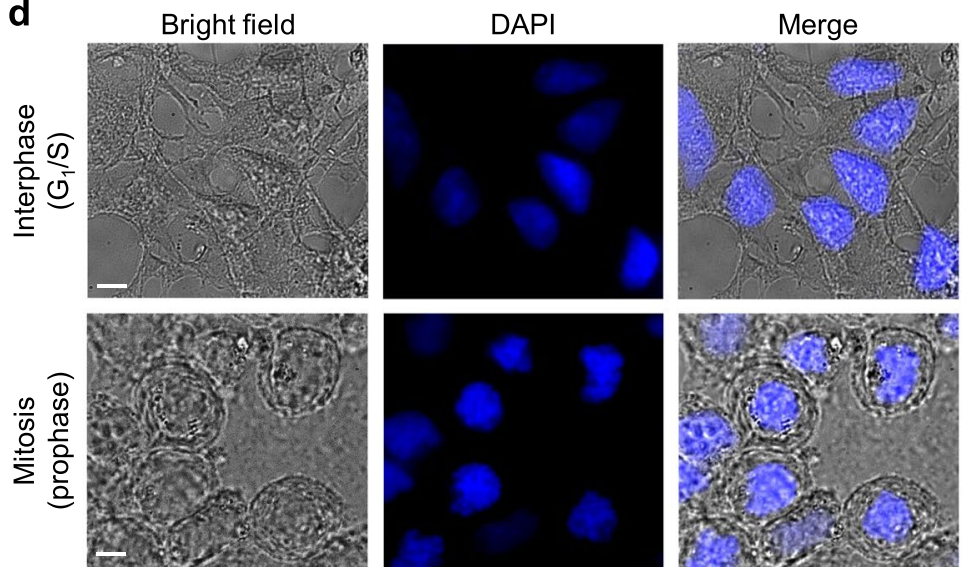

Figure 1. Analysis of HEK293 cell cultures by means of flow cytometry and conventional optical microscopy. $(\mathbf{a}-\mathbf{c})$ Flow cytometry histograms of asynchronous, interphase $\left(\mathrm{G}_{1} / \mathrm{S}\right)$ and mitosis (prophase) populations. Percentages correspond to cells in each cycle phase. (d) Bright-field and fluorescence microscopy images of the same interphase and mitosis populations. Scale bar, $10 \mu \mathrm{m}$.

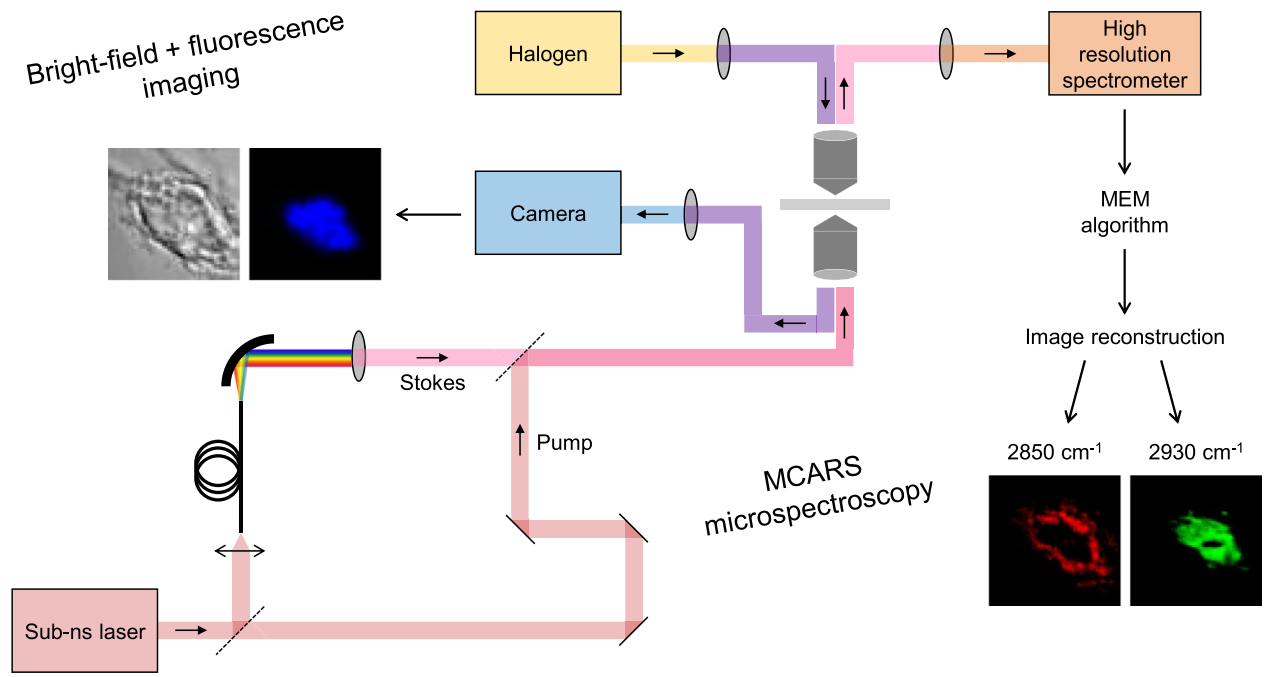

Figure 2. Experimental and numerical methodology for the analysis of cell cultures. First, cells of interest were selected using bright-field and/or fluorescence imaging. Then selected cells were mapped by means of high spectral resolution $\left(<1 \mathrm{~cm}^{-1}\right)$ MCARS microspectroscopy in the $2500-3200 \mathrm{~cm}^{-1}$ range (see Methods for details of the MCARS system). Finally, the vibrational information was extracted by using MEM algorithm, and cell images were reconstructed at $2850 \mathrm{~cm}^{-1}\left(\mathrm{CH}_{2}\right.$ symmetric stretching $)$ and $2930 \mathrm{~cm}^{-1}\left(\mathrm{CH}_{3}\right.$ symmetric stretching). 
a
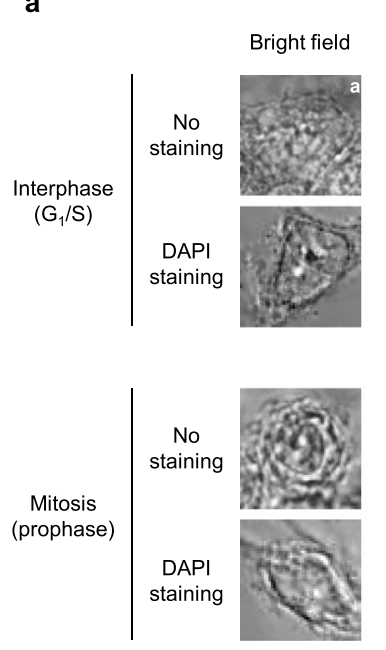
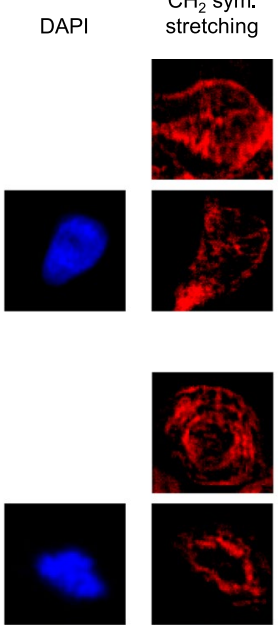
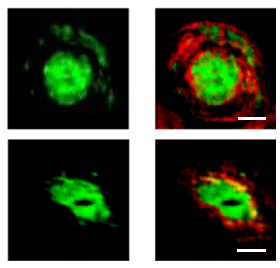

$\mathrm{CH}_{3}$ sym.

stretching
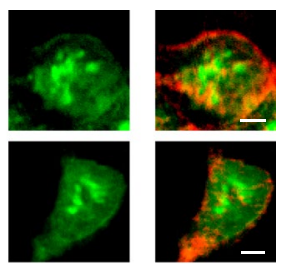

$\mathrm{CH}_{2} / \mathrm{CH}_{3}$
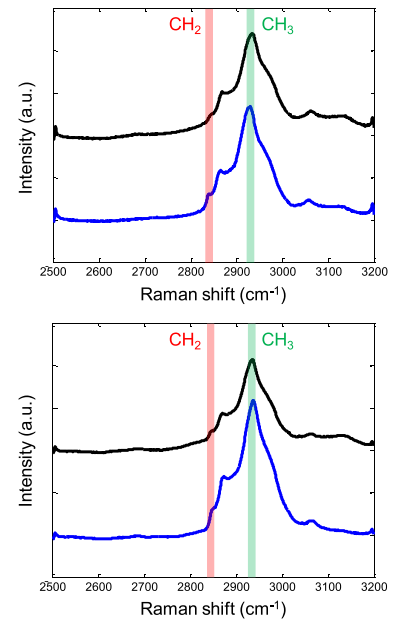

Figure 3. Analysis of interphase and mitotic, stained and unstained, fixed HEK293 cells. (a) Bright-field, fluorescence and MCARS $\left(\mathrm{CH}_{2}, \mathrm{CH}_{3}\right.$, merge) images of selected cells. MCARS images were reconstructed at $2850 \mathrm{~cm}^{-1}\left(\mathrm{CH}_{2}\right.$ symmetric stretching) and $2930 \mathrm{~cm}^{-1}\left(\mathrm{CH}_{3}\right.$ symmetric stretching). Scale bar, $5 \mu \mathrm{m}$. (b) Standard deviation of the vibrationally resonant CARS signal between 2500 and $3200 \mathrm{~cm}^{-1}$, computed over the whole analysis window of each cell. Spectra are plotted vertically in the same order as corresponding cells. $\mathrm{CH}_{2}$ and $\mathrm{CH}_{3}$ channels are highlighted.

We investigated interphase and mitotic, stained and unstained, HEK293 cells. Figure 3a shows the images obtained in each case. On the whole, $\mathrm{CH}_{2}$ and $\mathrm{CH}_{3}$ vibrational signatures do not overlap and thus bring complementary information from the sample. The $\mathrm{CH}_{2}$ signal - highlighting lipids - is mainly localised in the cytoplasm, when the $\mathrm{CH}_{3}$ signal - primarily associated with proteins - appears predominantly in the nucleus. The difference in $\mathrm{CH}_{2}$ signal distribution between interphase and mitosis would indicate the specific organization of the endoplasmic reticulum (ER) during these phases and will be discussed subsequently. Regarding the $\mathrm{CH}_{3}$ signal, bright spots corresponding to nucleoli are observed inside the nucleus of interphase cells, while the signal extends more homogeneously to the nucleus in mitotic cells. These findings are similar for stained and unstained cells, since the Raman signature of DAPI labelling is expected in the fingerprint region ${ }^{30,31}$. To confirm this, we computed the standard deviation of the vibrationally resonant CARS signal between 2500 and $3200 \mathrm{~cm}^{-1}$ over the whole analysis window of each cell, as plotted in Fig. 3b. The standard deviation spectra have the same profile in the $\mathrm{CH}$ stretching range, and no additional band arises because of DAPI staining. Thus, stained and unstained cells can be indifferently studied by MCARS microspectroscopy in the $2500-3200 \mathrm{~cm}^{-1}$ range.

Next, we quantified the contrast between $\mathrm{CH}_{2}$ and $\mathrm{CH}_{3}$ signal intensities in the nucleus for a set of 10 cells in interphase ( 5 stained, 5 unstained, see Supplementary Fig. 1) and 10 cells in mitosis ( 4 stained, 6 unstained, see Supplementary Fig. 2). For each cell, a region of interest (ROI) was drawn in the area of strong $\mathrm{CH}_{3}$ signature (Fig. 4, lower panel), then both $\mathrm{CH}_{2}$ and $\mathrm{CH}_{3}$ signal intensities were integrated in this ROI. Figure 4 (upper panel) displays the mean value of $\mathrm{CH}_{2}$ and $\mathrm{CH}_{3}$ integrated intensity for the set of interphase and mitotic cells. In either case, the intensity level of $\mathrm{CH}_{3}$ signature is significantly higher than that of $\mathrm{CH}_{2}$ signature. It is 3.10-fold higher than that of $\mathrm{CH}_{2}$ for interphase cells (6.55 \pm 1.62 and $2.11 \pm 0.65$, respectively), and 2.56-fold higher for mitotic cells ( $7.77 \pm 2.26$ and $3.04 \pm 0.87$, respectively). These results demonstrate that the biologically relevant complementarity between $\mathrm{CH}_{2}$ and $\mathrm{CH}_{3}$ vibrational signatures is obtained with high reproducibility. The $\mathrm{CH}_{2}$ stretching channel can reveal the ER organization in the cytoplasm. The $\mathrm{CH}_{3}$ one indicates the protein signal in the nucleus, highlighting nucleoli in particular.

Investigation of all sub-phases of mitosis. The aforementioned methodology was applied to the analysis of DAPI-stained HEK293 cells in the different sub-phases of mitosis (see Fig. 5). To identify each sub-phase easily, cells were observed by fluorescence microscopy before operating MCARS microspectroscopy. It is obvious that, in all sub-phases of mitosis, $\mathrm{CH}_{2}$ and $\mathrm{CH}_{3}$ signatures do not overlap, in agreement with previous observations. Interestingly, DAPI and $\mathrm{CH}_{3}$ signals show a significant colocalization, since the lateral shift between both images in some cases (prophase, prometaphase, metaphase) is clearly due to the different physical principles of these modalities. In other words, there is an uncertainty in the choice of the focal plane for MCARS imaging with respect to that used for fluorescence microscopy. Consistently, $\mathrm{CH}_{2}$ vibrational signal is predominant in the cytoplasm and does not overlap with the fluorescence signal of DAPI, whatever the sub-phase. The $\mathrm{CH}_{3}$ vibrational signature allows following the movement of chromosomes during the different sub-phases of mitosis; e.g., the alignment of chromosomes in metaphase, their separation in anaphase, or the formation of chromosome clusters at cell opposite poles in telophase.

Comparative analysis of fixed and living cells. Hoechst 33342 was used for staining the nucleus of living HEK293 cells, since it allows efficient labelling without cell permeabilization. We first investigated interphase 


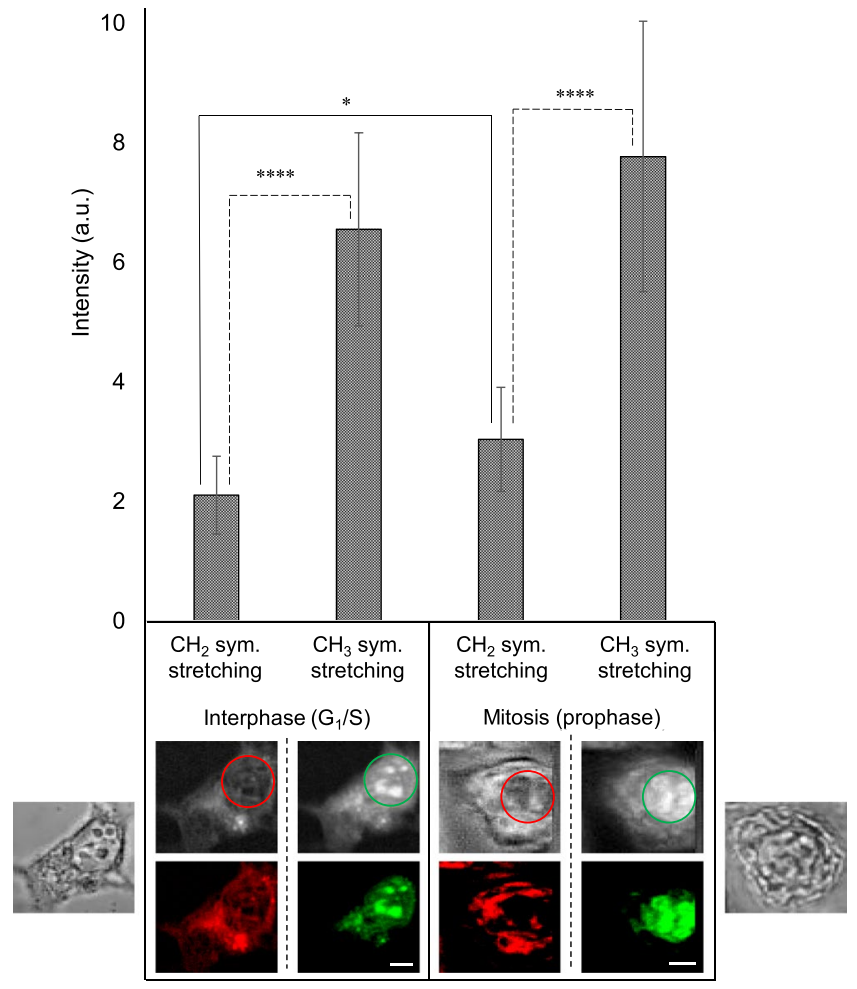

Figure 4. Quantification of the contrast between $\mathrm{CH}_{2}$ and $\mathrm{CH}_{3}$ signal intensities in the nucleus of fixed HEK293 cells. Lower panel represents bright-field and MCARS $\left(\mathrm{CH}_{2}, \mathrm{CH}_{3}\right)$ images of one interphase $\left(\mathrm{G}_{1} / \mathrm{S}\right)$ cell and one mitotic (prophase) cell. For each cell, an ROI was drawn in the area of strong $\mathrm{CH}_{3}$ signature, then $\mathrm{CH}_{2}$ and $\mathrm{CH}_{3}$ signal intensities were integrated in this ROI. This method was applied to 10 cells in interphase ( 5 stained, 5 unstained, see Supplementary Fig. 1) and 10 cells in mitosis ( 4 stained, 6 unstained, see Supplementary Fig. 2), throughout three independent experiments. Scale bar, $5 \mu \mathrm{m}$. Upper panel displays the mean value of $\mathrm{CH}_{2}$ and $\mathrm{CH}_{3}$ integrated intensity for this set of interphase and mitotic cells.

$\left(\mathrm{G}_{1} / \mathrm{S}\right)$, stained and unstained, living cells so as to assess the possible impact of Hoechst labelling on the vibrational signature in the $\mathrm{CH}$ stretching region. Actually, this fluorophore is expected to have specific Raman bands in the fingerprint region ${ }^{32}$, but its contribution in the high wavenumber region, even weak, must be considered ${ }^{33}$. Figure 6a shows bright-field, fluorescence and MCARS images obtained for two unstained (upper panel) and two stained (lower panel) cells. As previously, $\mathrm{CH}_{2}$ and $\mathrm{CH}_{3}$ signatures are complementary. Two particular features can be observed in the $\mathrm{CH}_{3}$ channel for both stained and unstained cells, namely the presence of bright intranuclear structures and the visualisation of nucleus border. Based on the images of Hoechst fluorescence, this corresponds to nucleoli and to heterochromatin contiguous to the nuclear envelop, respectively. Therefore, stained living cells can be accurately analysed by MCARS microspectroscopy in the $2500-3200 \mathrm{~cm}^{-1}$ range, since Hoechst labelling does not have any noteworthy effect on the vibrational signature. This assumption was corroborated by computing the standard deviation spectra of the vibrationally resonant CARS signal (Fig. 6b), which did not show substantial features due to Hoechst staining.

Then, we could compare fixed and living cells in the presence of DAPI and Hoechst staining, respectively. This study was realised for HEK293 cells and fibroblasts in interphase $\left(\mathrm{G}_{1} / \mathrm{S}\right)$ and mitosis (prophase), as presented in Fig. 7. In interphase, nucleoli are visible in the $\mathrm{CH}_{3}$ channel for both fixed and living cells, but the nucleus border is visible in the case of living cells only. This last observation was verified for a set of 10 fixed interphase cells (see Supplementary Fig. 1, where no nucleus border is visible) and 5 living interphase cells (see Figs 6a and 7, where the nucleus border is noticeable). Such phenomenon is therefore related to the cell fixation process and could be probed by means of MCARS microspectroscopy. In mitosis, only chromosomes are observed for both fixed and living cells, as expected.

\section{Discussion}

In this work, we apply MCARS microspectroscopy in the $\mathrm{CH}$ stretching vibrational region $\left(2500-3200 \mathrm{~cm}^{-1}\right)$ to the analysis of a human cell line during the cell cycle. Generally, to observe the nucleus by vibrational technique through its DNA content, authors use the fingerprint region with Raman shifts at (1) $785 \mathrm{~cm}^{-1}$ corresponding to uracil/thymine ring breathing ${ }^{2,6}$, (2) $1095 \mathrm{~cm}^{-1}$ corresponding to phosphate symmetric stretching in the DNA backbone $e^{8,19,34}$, (3) $1487 \mathrm{~cm}^{-1}$ and $1578 \mathrm{~cm}^{-1}$ corresponding to adenine/guanine ring breathing, respectively ${ }^{19,34}$. Another strategy consists in focusing on proteins, which are the main component of heterochromatin (they represent two thirds of its mass). So, in view of the quantity of proteins present in heterochromatin, the analysis in 


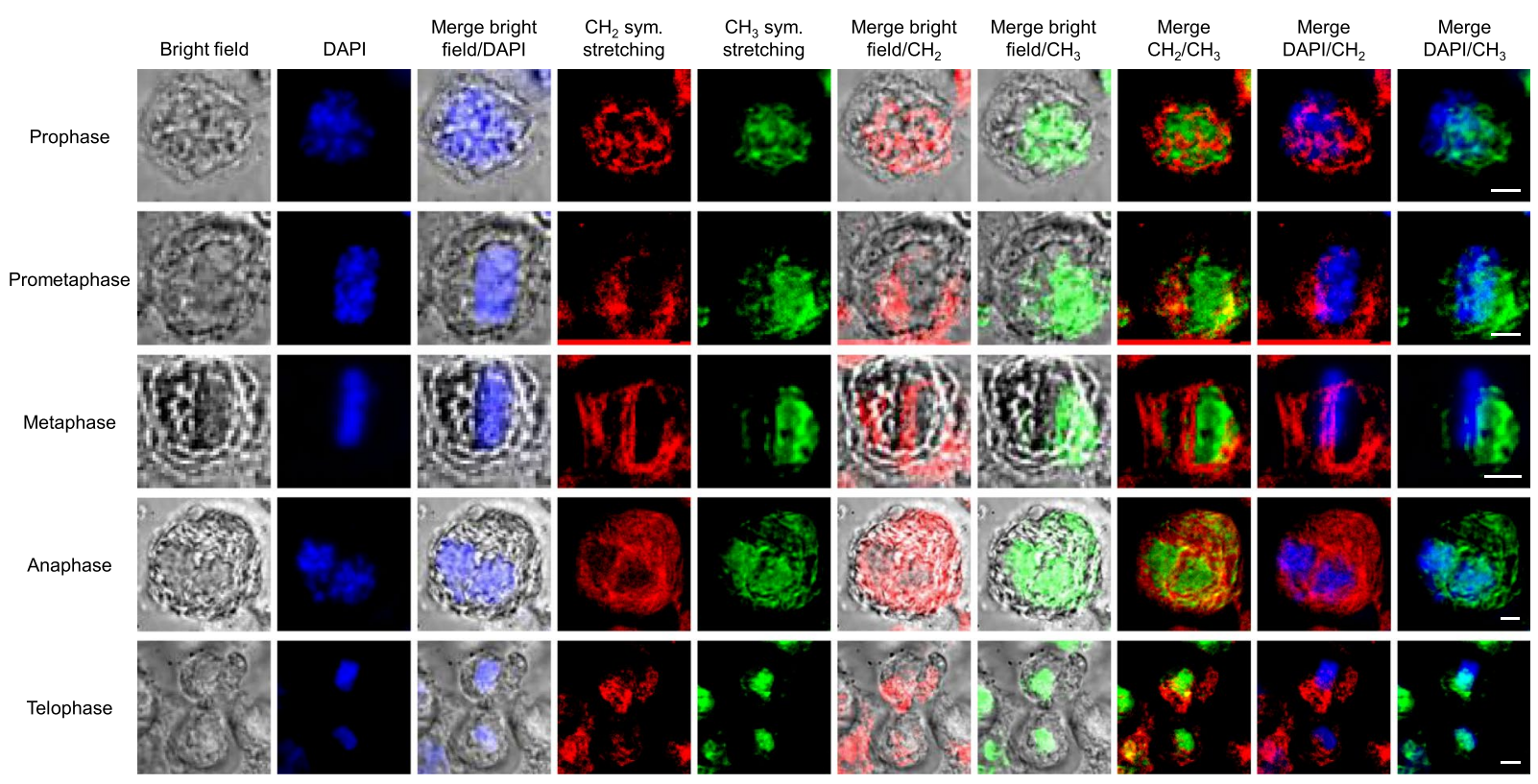

Figure 5. Analysis of DAPI-stained HEK293 cells in the different sub-phases of mitosis, including bright-field, fluorescence and MCARS $\left(\mathrm{CH}_{2}, \mathrm{CH}_{3}\right)$ imaging. Scale bar, $5 \mu \mathrm{m}$.

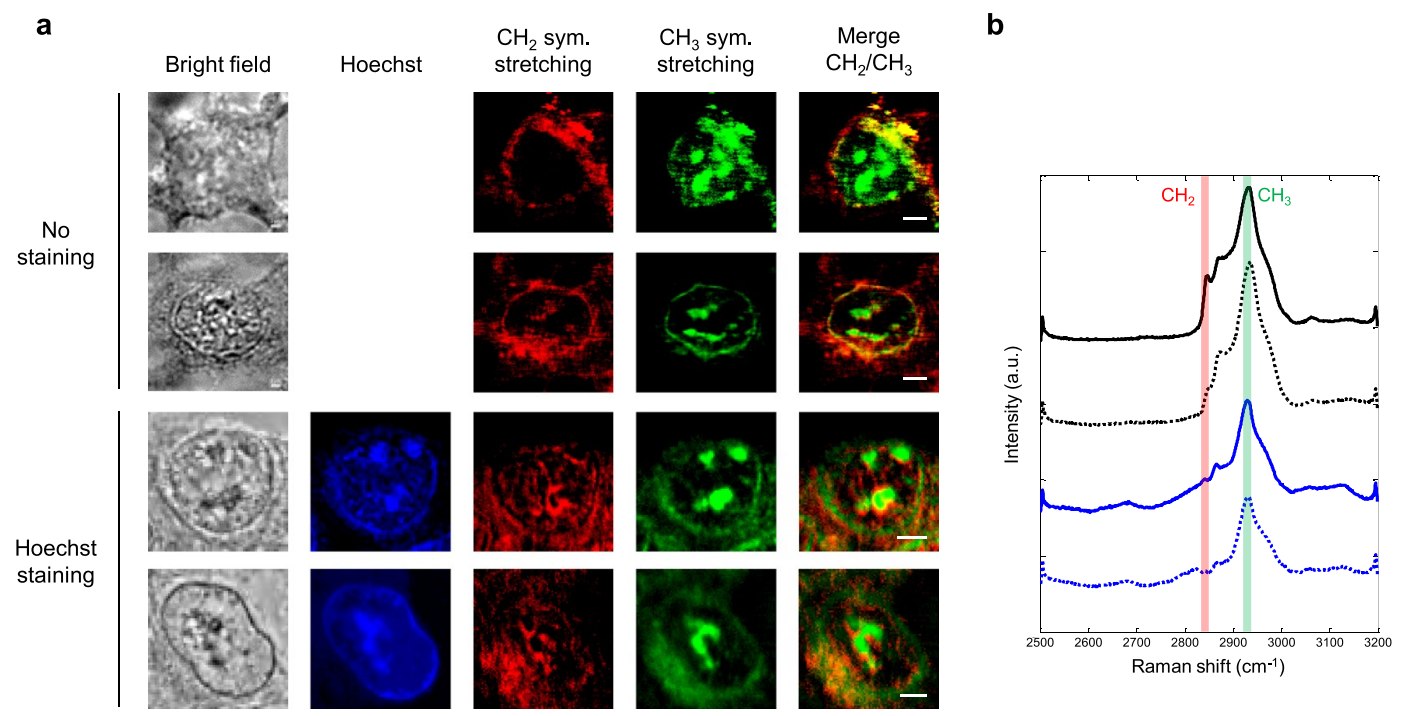

Figure 6. Analysis of interphase $\left(\mathrm{G}_{1} / \mathrm{S}\right)$, stained and unstained, living HEK 293 cells. (a) Bright-field, fluorescence and MCARS $\left(\mathrm{CH}_{2}, \mathrm{CH}_{3}\right.$, merge) images of selected cells. MCARS images were reconstructed at $2850 \mathrm{~cm}^{-1}\left(\mathrm{CH}_{2}\right.$ symmetric stretching $)$ and $2930 \mathrm{~cm}^{-1}\left(\mathrm{CH}_{3}\right.$ symmetric stretching $)$. Scale bar, $5 \mu \mathrm{m}$. (b) Standard deviation of the vibrationally resonant CARS signal between 2500 and $3200 \mathrm{~cm}^{-1}$, computed over the whole analysis window of each cell. Spectra are plotted vertically in the same order as corresponding cells. $\mathrm{CH}_{2}$ and $\mathrm{CH}_{3}$ channels are highlighted.

the $\mathrm{CH}$ region is expected to provide stronger vibrational signatures compared to the fingerprint region, in favour of obtaining high contrast CARS images.

The difference in $\mathrm{CH}_{3}$ signature intensity between mitotic (prophase) and interphase $\left(\mathrm{G}_{1} / \mathrm{S}\right)$ cells (Fig. 4$)$ may be due to the condensation state of chromatin: a strong condensation during mitosis (only heterochromatin) which requires a large amount of proteins such as histones, condensins and cohesins; and a lower condensation in interphase during which heterochromatin represents only a part of the whole chromatin in nucleus, mainly in nucleoli ${ }^{35}$. In this case, the rest of DNA is packaged in euchromatin, which is less condensed and includes a small amount of proteins, mostly histones. Besides, for interphase fixed (Figs 3 and 4) and living (Figs 6 and 7) cells, heterochromatin is depicted in the $\mathrm{CH}_{3}$ stretching channel as the nucleolar structures, corresponding to the bright spots observed in the nucleus with DAPI or Hoechst fluorescence, respectively. We cannot exclude that 


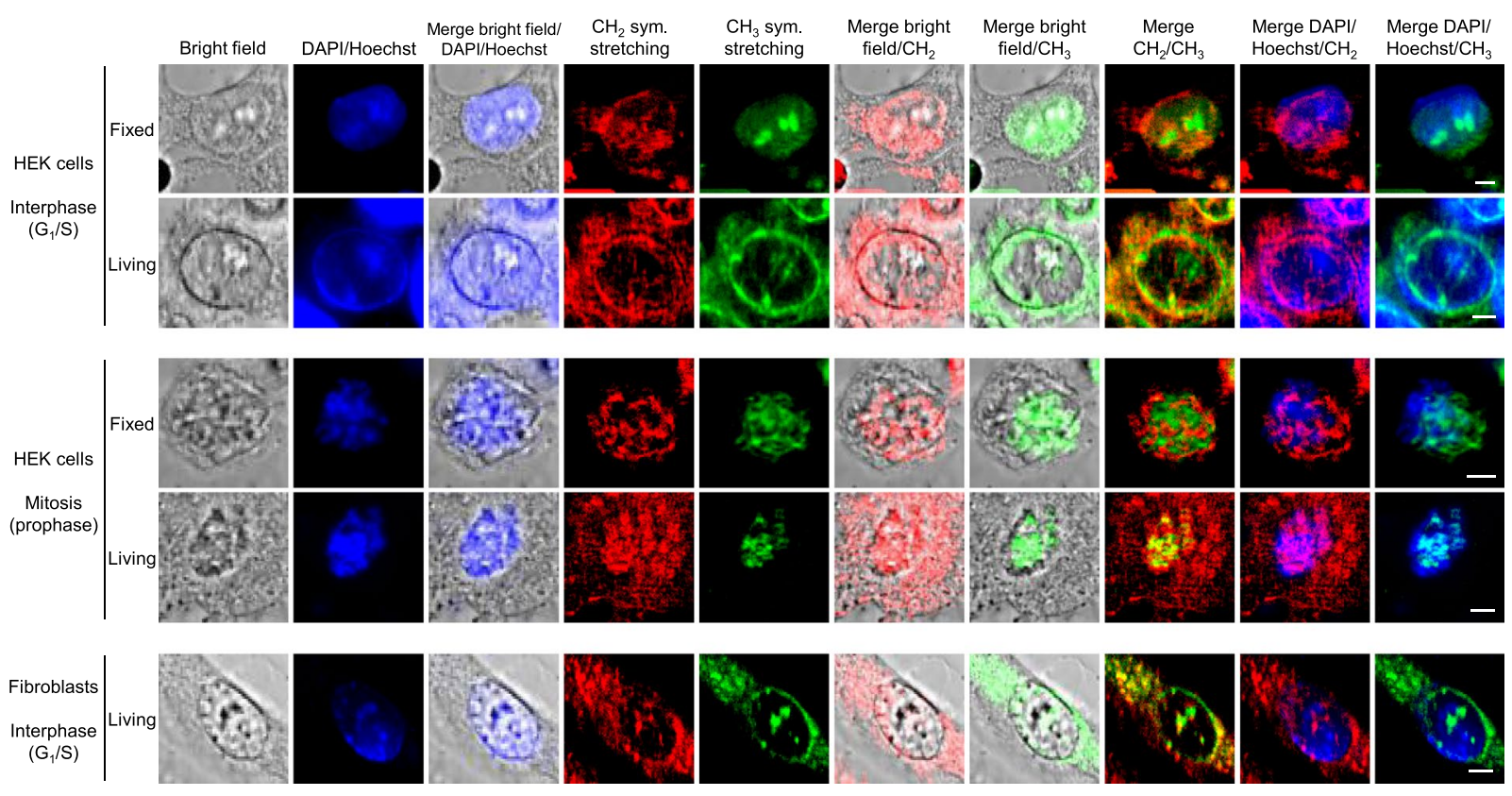

Figure 7. Analysis of interphase and mitotic, stained, fixed and living, HEK293 cells and fibroblasts, including bright-field, fluorescence and MCARS $\left(\mathrm{CH}_{2}, \mathrm{CH}_{3}\right)$ imaging. Fixed and living HEK293 cells were stained with DAPI and Hoechst 33342, respectively. Living fibroblasts were stained with Hoechst 33342. Scale bar, $5 \mu \mathrm{m}$.

$\mathrm{CH}_{3}$ stretching at $2930 \mathrm{~cm}^{-1}$ corresponds mainly to proteins. It may also include a signal due to the presence of 5 -metylcytosine, which is a hallmark of heterochromatin ${ }^{36}$.

Regarding the $\mathrm{CH}_{2}$ vibrational signature, it is primarily associated to lipids and therefore to plasma and organelle membranes. During the cell cycle, there is a remodelling and reorganization of the organelles in the cytoplasm. Among them, the ER corresponds to a large part of endogenous membranes. During interphase, the ER displays a typical reticular network of cisternae (sheets) and tubules, but it is predominantly organised as extended cisternae during mitosis ${ }^{37}$. This cisternal organisation of the ER is obvious for unstained prophase cells of Supplementary Fig. 2, indicating the effectiveness of MCARS microspectroscopy for probing the specific ER morphology during mitosis. Additionally, we could observe that the ER accumulates at the spindle poles and is excluded from the central area in prometaphase/metaphase ${ }^{38}$ : this phenomenon is clearly visualised in the $\mathrm{CH}_{2}$ stretching channel for the prometaphase cell of Fig. 5.

We now discuss the impact of cell fixation on the visualisation of the nucleus border. For living cells only, the border of the nucleus is clearly visible through $\mathrm{CH}_{3}$ vibrational signature (Figs 6 and 7). This may be explained by the presence of heterochromatin, which binds indirectly to the nuclear envelope by means of proteins involved in tethering chromatin during interphase. Many nuclear envelope transmembrane proteins (NETs) binding chromatin have been identified, such as MAN1, LAP2 $\beta$ or emerin. They interact with chromatin proteins or methylated histones, and also with nuclear lamina ${ }^{39,40}$. Therefore, heterochromatin is retained at the inner nuclear membrane and allows to delimitate the nucleus in cell through its $\mathrm{CH}_{3}$ vibrational signature. When cells are fixed with PFA, the nuclear envelope is biochemically modified. The treatment may induce a loss of interaction between the NETs and the heterochromatin, which is no more connected to the inner nuclear membrane and may be released in the nucleoplasm. Only nucleoli, which contain a high quantity of heterochromatin, remain observable. These results are relevant in view of the numerous studies of cells fixed by PFA based on vibrational technologies.

For the simultaneous observation of chromosome movement during mitosis and of other structures in cell such as ER by epifluorescence, confocal, or two-photon microscopy, it is necessary to label DNA with specific dyes and cell compartment with specific antibodies. In both cases, cells must be permeabilized. Specific cell compartment labelling often requires the use of a primary antibody associated to a secondary one coupled to a fluorochrome. We show that MCARS microspectroscopy allows to directly visualize different cell components (chromosomes, ER) without labelling and cell permeabilization.

This work shows that high spectral resolution MCARS microspectroscopy in the high wavenumber region allows to visualise proliferation, the most common cellular process, with no prior labelling. By means of the vibrational signature of condensation proteins, structures like nucleoli, nuclear border and chromosomes can be identified. Furthermore, the structuration of the cytoplasm and especially the ER organisation can be effectively probed through the signature of lipids. It is also stressed that MCARS can reveal the impact of the fixative method on these structures. With this label-free imaging approach, it will be possible to visualize other cellular processes for which chromatin undergoes rearrangements. 


\begin{abstract}
Methods
Cell culture. Human embryonic kidney 293 (HEK293) cells were cultured in a DMEM medium $(4.5 \mathrm{~g} / \mathrm{L}$ of glucose, Gibco) supplemented with $10 \%$ (v/v) fetal calf serum (BioWest), 100 units $/ \mathrm{mL}$ penicillin and $100 \mu \mathrm{g} / \mathrm{mL}$ streptomycin (Gibco) at $37^{\circ} \mathrm{C}$ under humidified atmosphere and $5 \% \mathrm{CO}_{2}$.

Fibroblast primary culture was seeded in a DMEM medium $(4.5 \mathrm{~g} / \mathrm{L}$ of glucose, Gibco) supplemented with amphotericin B at $1 \mu \mathrm{g} / \mathrm{mL}$ (Gibco), 100 units/mL penicillin and $100 \mu \mathrm{g} / \mathrm{mL}$ streptomycin and $10 \%$ (v/v) fetal calf serum at $37^{\circ} \mathrm{C}$ under humidified atmosphere and $5 \% \mathrm{CO}_{2}$.

HEK293 cells and fibroblasts were grown on uncoated glass slides to prevent generation of vibrational signal from the holder during the upcoming MCARS analysis. We used 12-well plates in which we introduced $18 \mathrm{~mm}$ diameter round glass coverslips. Cells were seeded at a density of 35.000 cells per well.
\end{abstract}

Cell synchronization. To obtain populations of cells at $\mathrm{G}_{1} / \mathrm{S}$ boundary, we used a double thymidine block, since thymidine is inhibitor of DNA synthesis. After $48 \mathrm{~h}$ of culture in 12 -well plate, cells were treated with thymidine at $2 \mathrm{mM}$ during $18 \mathrm{~h}$, released for $9 \mathrm{~h}$ by washing out the thymidine and then blocked again by addition in the culture medium of $2 \mathrm{mM}$ of thymidine for $17 \mathrm{~h}^{41}$.

Concerning cell arrest in mitosis (prophase), after $48 \mathrm{~h}$ of culture, cells were treated with thymidine at $2 \mathrm{mM}$ during $18 \mathrm{~h}$. Then, cells were washed in DPBS (Dulbecco's phosphate-buffered saline) and released for $3 \mathrm{~h}$ in complete medium allowing to enter $\mathrm{S}$ phase. Finally, cells were blocked with nocodazole at $100 \mathrm{ng} / \mathrm{mL}$ during $12 \mathrm{~h}^{41}$.

To analyse the different sub-phases of mitosis, cells were blocked in prophase as described above. After washing with DPBS to remove nocodazole, $1 \mathrm{~mL}$ of complete culture medium was added to the wells. Therefore, cells could advance synchronously in mitosis. Every $30 \mathrm{~min}$, cells were washed and fixed.

Cell fixation and staining. Unlabelled fixed cells. After synchronization, cells were washed three times with DPBS and fixed by PFA $4 \%$ (v/v) in DPBS during $10 \mathrm{~min}$ at room temperature. After three washings to eliminate PFA, the coverslip was sealed with nail polish on a microscopy glass slide.

Fixed cells with DAPI staining. After fixation and washing, the nuclei were labelled with 4',6-diamidino-2-phenylindole (DAPI, Sigma-Aldrich) at $1 \mu \mathrm{g} / \mathrm{mL}$ for $5 \mathrm{~min}$. Then the cells were washed, and the coverslip sealed as previously.

Living cells with Hoechst staining. Hoechst 33342 (Thermo Fisher Scientific) at $10 \mu \mathrm{g} / \mathrm{mL}$ was used during $15 \mathrm{~min}$ for staining living cells. Then the cells were washed, and the coverslip sealed as previously.

Flow cytometry. Flow cytometry analysis was realized before (as a negative control) and after cell synchronization with $10^{6}$ cells. After centrifugation at $1500 \mathrm{RPM}$, the pellet was suspended in $300 \mu \mathrm{L}$ of cold PBS and rapidly in $700 \mu \mathrm{L}$ of cold ethanol (100\%). The cell suspension was preserved at $-20^{\circ} \mathrm{C}$ overnight. Then cells were washed twice with DPBS, re-suspended with $1 \mathrm{~mL}$ of DPBS and treated with RNase A (Sigma-Aldrich) at $1 \mu \mathrm{g} / \mathrm{mL}$ for $20 \mathrm{~min}$ at room temperature. After that, cells were labelled with propidium iodide $(0.5 \mathrm{mg} / \mathrm{mL})$ and immediately analysed by means of FACSCalibur (Becton Dickinson) flow cytometer. The resulting cell distributions were computed by Modfit software in order to determine the proportion of cells in the different phases of the cell cycle.

Fluorescence microscopy. Stained cells were observed with an epifluorescence microscope (Leica DMI4000B). Data were processed using MetaMorph software (Molecular Devices).

MCARS microspectroscopy. The MCARS system is an adaptation, in forward configuration, of our epi-detected custom-built setup ${ }^{42}$. It is designed as follows. The pump source is a passively Q-switched microchip laser (Horus Laser, $1064 \mathrm{~nm}, 1 \mathrm{~ns}, 20 \mathrm{kHz}$, linearly polarized, $<0.1 \mathrm{~cm}^{-1}$ linewidth). The laser beam is divided into two parts by using a half-wave plate and a Glan-Taylor polarizer. One part is injected into a photonic crystal fibre to generate a supercontinuum Stokes wave $(600-1650 \mathrm{~nm})$. This Stokes beam is collimated by means of a parabolic mirror and directed to a long-pass filter at $1050 \mathrm{~nm}$ (Thorlabs, FEL1050). The other part is used as the pump radiation of the CARS process after adjusting its power with a variable neutral density filter and its delay with a delay line. Then pump and Stokes beams are spatially combined through a notch dichroic beamsplitter (Semrock, NFD01-1064-25 $\times 36$ ) and tightly focused onto the sample with a high numerical aperture microscope objective (Olympus, UPlanSApo 60x, N.A.=1.2, water immersion). The position of the sample is controlled by means of a translation stage. The CARS signal generated by the sample is collected by a second microscope objective (Nikon, S Plan Fluor ELWD 60x, N.A. = 0.7), cleaned from any remaining pump signal by using a notch filter (Thorlabs, NF1064-44) and guided into a spectrometer (Horiba, LabRam HR Evolution, $600 \mathrm{gr} / \mathrm{mm}$ grating, Synapse CCD camera). In order to observe stained cells via the fluorescence of DAPI or Hoechst, the M-CARS system was complemented with a halogen light source, appropriate excitation and emission filters, and a dedicated CCD camera (Thorlabs, 1500M-GE).

The lateral, axial and spectral resolutions of the CARS microspectroscope are $\sim 300 \mathrm{~nm}, 2 \mu \mathrm{m}$ and $0.8 \mathrm{~cm}^{-1}$ respectively. During all experiments, the laser power of pump and Stokes radiations at the sample position was set at $55 \mathrm{~mW}$ and $9 \mathrm{~mW}$, respectively. At this laser power (64 mW in total), no morphological change of cells was observed. This was confirmed by visualizing the sample with bright-field and/or fluorescence imaging.

After being selected by means of bright-field and/or fluorescence imaging, cells were analysed by MCARS microspectroscopy, using a lateral step of $300 \mathrm{~nm}$ for the cross-section mapping. Spectra were acquired from 2500 to $3200 \mathrm{~cm}^{-1}$ with $50 \mathrm{~ms}$ pixel dwell time and processed by using the MEM so as to extract the pure vibrationally resonant signal, which corresponds to the imaginary part of the third order nonlinear susceptibility $\left(\operatorname{Im}\left\{\chi^{(3)}\right\}\right)^{29}$. 


\section{Data Availability}

The datasets generated during the current study are available from corresponding author P.L. (philippe.leproux@ unilim.fr) on reasonable request.

\section{References}

1. van Manen, H.-J., Kraan, Y. M., Roos, D. \& Otto, C. Single-cell Raman and fluorescence microscopy reveal the association of lipid bodies with phagosomes in leukocytes. Proceedings of the National Academy of Sciences 102, 10159-10164 (2005).

2. Matthäus, C., Chernenko, T., Newmark, J. A., Warner, C. M. \& Diem, M. Label-Free Detection of Mitochondrial Distribution in Cells by Nonresonant Raman Microspectroscopy. Biophysical Journal 93, 668-673 (2007).

3. Scalfi-Happ, C., Udart, M., Hauser, C. \& Rück, A. Investigation of lipid bodies in a colon carcinoma cell line by confocal Raman microscopy. Medical Laser Application 26, 152-157 (2011).

4. Abramczyk, H. et al. The role of lipid droplets and adipocytes in cancer. Raman imaging of cell cultures: MCF10A, MCF7, and MDA-MB-231 compared to adipocytes in cancerous human breast tissue. The Analyst 140, 2224-2235 (2015).

5. Mariani, M. M. et al. Micro-Raman Detection of Nuclear Membrane Lipid Fluctuations in Senescent Epithelial Breast Cancer Cells. Analytical Chemistry 82, 4259-4263 (2010).

6. Kang, J. et al. Investigating Effects of Proteasome Inhibitor on Multiple Myeloma Cells Using Confocal Raman Microscopy. Sensors 16, $2133(2016)$

7. Zumbusch, A., Holtom, G. R. \& Xie, X. S. Three-Dimensional Vibrational Imaging by Coherent Anti-Stokes Raman Scattering. Physical Review Letters 82, 4142-4145 (1999).

8. Cheng, J.-X., Jia, Y. K., Zheng, G. \& Xie, X. S. Laser-Scanning Coherent Anti-Stokes Raman Scattering Microscopy and Applications to Cell Biology. Biophysical Journal 83, 502-509 (2002).

9. Moura, C. C., Tare, R. S., Oreffo, R. O. C. \& Mahajan, S. Raman spectroscopy and coherent anti-Stokes Raman scattering imaging: prospective tools for monitoring skeletal cells and skeletal regeneration. Journal of The Royal Society Interface 13, 20160182 (2016).

10. Weeks, T., Schie, I., den Hartigh, L. J., Rutledge, J. C. \& Huser, T. Lipid-cell interactions in human monocytes investigated by doublyresonant coherent anti-Stokes Raman scattering microscopy. Journal of Biomedical Optics 16, 021117 (2011).

11. Nan, X., Cheng, J.-X. \& Xie, X. S. Vibrational imaging of lipid droplets in live fibroblast cells with coherent anti-Stokes Raman scattering microscopy. Journal of Lipid Research 44, 2202-2208 (2003).

12. Lee, Y. J. et al. Quantitative, Label-Free Characterization of Stem Cell Differentiation at the Single-Cell Level by Broadband Coherent Anti-Stokes Raman Scattering Microscopy. Tissue Engineering Part C: Methods 20, $562-569$ (2014).

13. Le, T. T., Huff, T. B. \& Cheng, J.-X. Coherent anti-Stokes Raman scattering imaging of lipids in cancer metastasis. BMC Cancer 9 (2009).

14. Mitra, R., Chao, O., Urasaki, Y., Goodman, O. B. \& Le, T. T. Detection of Lipid-Rich Prostate Circulating Tumour Cells with Coherent Anti-Stokes Raman Scattering Microscopy. BMC Cancer 12 (2012).

15. Rodriguez, L. G., Lockett, S. J. \& Holtom, G. R. Coherent anti-stokes Raman scattering microscopy: A biological review. Cytometry Part A 69A, 779-791 (2006).

16. Leproux, P. et al. New opportunities offered by compact sub-nanosecond supercontinuum sources in ultra-broadband multiplex CARS microspectroscopy. Journal of Raman Spectroscopy 42, 1871-1874 (2011).

17. Okuno, M., Kano, H., Leproux, P., Couderc, V. \& Hamaguchi, H. Ultrabroadband multiplex CARS microspectroscopy and imaging using a subnanosecond supercontinuum light source in the deep near infrared. Optics Letters 33, 923 (2008).

18. Okuno, M. et al. Quantitative CARS Molecular Fingerprinting of Single Living Cells with the Use of the Maximum Entropy Method. Angewandte Chemie International Edition 49, 6773-6777 (2010).

19. Parekh, S. H., Lee, Y. J., Aamer, K. A. \& Cicerone, M. T. Label-Free Cellular Imaging by Broadband Coherent Anti-Stokes Raman Scattering Microscopy. Biophysical Journal 99, 2695-2704 (2010).

20. Camp, C. H. Jr. et al. High-speed coherent Raman fingerprint imaging of biological tissues. Nature Photonics 8, 627-634 (2014).

21. Czamara, K. et al. Impact of cell cycle dynamics on pathology recognition: Raman imaging study. Journal of Biophotonics 12, e201800152 (2019).

22. Harper, J. V. \& Brooks, G. The Mammalian Cell Cycle: An Overview. in Cell Cycle Control 296, 113-154 (Humana Press, 2004).

23. Matthäus, C., Boydston-White, S., Miljković, M., Romeo, M. \& Diem, M. Raman and Infrared Microspectral Imaging of Mitotic Cells. Applied Spectroscopy 60, 1-8 (2006).

24. Pliss, A., Kuzmin, A. N., Kachynski, A. V. \& Prasad, P. N. Nonlinear Optical Imaging and Raman Microspectrometry of the Cell Nucleus throughout the Cell Cycle. Biophysical Journal 99, 3483-3491 (2010).

25. Karuna, A. et al. Label-Free Volumetric Quantitative Imaging of the Human Somatic Cell Division by Hyperspectral Coherent AntiStokes Raman Scattering. Analytical Chemistry 91, 2813-2821 (2019).

26. Lu, F.-K. et al. Label-free DNA imaging in vivo with stimulated Raman scattering microscopy. Proceedings of the National Academy of Sciences 112, 11624-11629 (2015)

27. Yoneyama, H. et al. Invited Article: CARS molecular fingerprinting using sub-100-ps microchip laser source with fiber amplifier. APL Photonics 3, 092408 (2018).

28. Cadart, C., Zlotek-Zlotkiewicz, E., Le Berre, M., Piel, M. \& Matthews, H. K. Exploring the Function of Cell Shape and Size during Mitosis. Developmental Cell 29, 159-169 (2014).

29. Vartiainen, E. M., Rinia, H. A., Müller, M. \& Bonn, M. Direct extraction of Raman line-shapes from congested CARS spectra. Optics Express 14, $3622(2006)$.

30. Dou, X. et al. Quantitative analysis of double-stranded DNA amplified by a polymerase chain reaction employing surface-enhanced Raman spectroscopy. Applied Optics 37, 759 (1998).

31. Krause, M., Radt, B., Rösch, P. \& Popp, J. The investigation of single bacteria by means of fluorescence staining and Raman spectroscopy. Journal of Raman Spectroscopy 38, 369-372 (2007).

32. Uzunbajakava, N. \& Otto, C. Combined Raman and continuous-wave-excited two-photon fluorescence cell imaging. Optics Letters 28, 2073 (2003)

33. Pully, V. V., Lenferink, A. \& Otto, C. Hybrid Rayleigh, Raman and two-photon excited fluorescence spectral confocal microscopy of living cells. Journal of Raman Spectroscopy 41, 599-608 (2009).

34. Matthews, Q., Jirasek, A., Lum, J., Duan, X. \& Brolo, A. G. Variability in Raman Spectra of Single Human Tumor Cells Cultured in vitro: Correlation with Cell Cycle and Culture Confluency. Applied Spectroscopy 64, 871-887 (2010).

35. Lam, Y. W. The nucleolus. Journal of Cell Science 118, 1335-1337 (2005).

36. Lewis, J. \& Bird, A. DNA methylation and chromatin structure. FEBS Letters 285, 155-159 (1991).

37. Lu, L., Ladinsky, M. S. \& Kirchhausen, T. Cisternal Organization of the Endoplasmic Reticulum during Mitosis. Molecular Biology of the Cell 20, 3471-3480 (2009).

38. Schlaitz, A.-L. Microtubules as key coordinators of nuclear envelope and endoplasmic reticulum dynamics during mitosis: Prospects \& Overviews. BioEssays 36, 665-671 (2014).

39. Poleshko, A. \& Katz, R. A. Specifying peripheral heterochromatin during nuclear lamina reassembly. Nucleus 5, 32-39 (2014).

40. Czapiewski, R., Robson, M. I. \& Schirmer, E. C. Anchoring a Leviathan: How the Nuclear Membrane Tethers the Genome. Frontiers in Genetics 7, 82 (2016). 
41. Whitfield, M. L. et al. Stem-Loop Binding Protein, the Protein That Binds the 3J End of Histone mRNA, Is Cell Cycle Regulated by Both Translational and Posttranslational Mechanisms. Molecular and Cellular Biology. 20, 4188-4198 (2000).

42. Capitaine, E. et al. Fast epi-detected broadband multiplex CARS and SHG imaging of mouse skull cells. Biomedical Optics Express 9 , 245 (2018).

\section{Author Contributions}

T.G.D.B. prepared the samples. Z.R. and P.L. developed the custom-made MCARS system. T.G.D.B. and P.L. performed the experiments and analysed the data. V.C., V.S. and H.K. contributed to discussions. J.M.P. and P.L. supervised and coordinated the project. T.G.D.B., J.M.P. and P.L. wrote the manuscript with contributions from all authors.

\section{Additional Information}

Supplementary information accompanies this paper at https://doi.org/10.1038/s41598-019-50453-0.

Competing Interests: The authors declare no competing interests.

Publisher's note Springer Nature remains neutral with regard to jurisdictional claims in published maps and institutional affiliations.

(c) (i) Open Access This article is licensed under a Creative Commons Attribution 4.0 International License, which permits use, sharing, adaptation, distribution and reproduction in any medium or format, as long as you give appropriate credit to the original author(s) and the source, provide a link to the Creative Commons license, and indicate if changes were made. The images or other third party material in this article are included in the article's Creative Commons license, unless indicated otherwise in a credit line to the material. If material is not included in the article's Creative Commons license and your intended use is not permitted by statutory regulation or exceeds the permitted use, you will need to obtain permission directly from the copyright holder. To view a copy of this license, visit http://creativecommons.org/licenses/by/4.0/.

(C) The Author(s) 2019 